\title{
NOT ONLY A CHARGE: INVOLVING STUDENTS IN THE OIL DROP EXPERIMENT
}

\author{
Roberto Mazzola ${ }^{1}$, Lucia Zucchelli ${ }^{1}$, Matteo Bozzi ${ }^{2,3}$, Maurizio Zani ${ }^{2}$ \\ ${ }^{1}$ Liceo Scientifico Statale "J. C. Maxwell", Milano (ITALY) \\ ${ }^{2}$ Department of Physics, Politecnico di Milano (ITALY) \\ ${ }^{3}$ Liceo Scientifico Statale "Vittorio Veneto", Milano (ITALY)
}

\begin{abstract}
An activity about the Millikan oil drop experiment is proposed to a group of 17-years-old students attending Liceo Scientifico opzione scienze applicate at the "IIS J. C. Maxwell" school located in Milan (Italy). The students were involved in some laboratory activities, cooperative working and whole class sessions guided by some teacher's questions. The classes are asked to be involved in the solution of a complex problem, starting from the experimental study of the motion of a body in a viscous fluid until facing the problem of the measurement of the charge some tiny oil drops immersed in an electric field.

The quantification of the quantum of electric charge was possible, and the students were first-hand dragged in a historical experiment. Primary goal of the activities is the improvement of skills such as the capacity of formulating hypotheses, the employment of an available instrumentation, the collection and analyses of data, the resolution of mathematical equation for the identification of physical quantities of interest.
\end{abstract}

A didactic video of the same experiment was produced by the Experimental teaching lab. $S T 2^{1}$ of Politecnico di Milano in order to foster the students' reflection on the same experience as a follow-up activity and, in an Open Educational Resources philosophy (OER), it will be made available on the web site of Lab. ST2 YouTube channel. Our project was developed in the context of Piano Nazionale Lauree Scientifiche (PLS), a government programme which funds didactic innovation of Science, Technology, Engineering and Mathematics (STEM) in Higher Education and Secondary School level.

Keywords: Learning effectiveness, Experimental video, Physics, Open Educational Resources

\section{INTRODUCTION}

The Italian government has recently reformed the examination which concludes the Secondary School study programme; with reference to Liceo Scientifico, i.e. a specific kind of High School with students specialising in scientific subjects, this reform has emphasised the crucial role played by Physics in this curriculum. As a consequence, a renewal in the teaching of Physics should be a primary aim. This change ought to increase the time and energies allotted to experimental didactic activities as well as the use of ad hoc educational videos on Physics phenomena [1], also facilitating a constructive dialogue between Upper Secondary Schools and Universities [2].

From this perspective, an interesting case is the quantum theory studied during the High School program and firstly introduced to students by presenting the elementary electric charge. The electric field is usually explained by comparison with the gravitational field. This comparison is rather useful and straightforward from a mathematical point of view, but it is experimentally less evident. In facts, the effects due to the gravitational uniform field are more easily comprehensible by students, whereas the effects of an electrical field are not so clear and measurable.

Although several qualitative experiments are usually carried out by teachers to explain electrical phenomena, quantitative studies are more difficult to be performed due to practical reasons and lack of adequate laboratory equipment in many schools. Among others, a meaningful quantitative experience to comprehend the electric field and its effects is the oil drop experiment performed by Millikan in 1905. Even though the core of his work was the measurement of the "quantum of charge", it involved many topics such as the motion within a viscous fluid, the electric uniform field and its force on a charge and the elementary charge.

This activity can help to reach many didactic purposes: among them we highlight these key points:

${ }^{1}$ http://www.st2.fisi.polimi.it 
- students involvement;

- learning a global overlook on the laws of physics;

- better comprehension and experimentation of the scientific method.

\section{METHODOLOGY}

The activity was proposed in two classes of the fourth year of the Liceo Scientifico and it is inserted at the end of a first introduction on electric phenomena. For instance some simple experiences can be performed in laboratory to show some electrostatic experiments such as the triboelectric effect and other modalities for the charging of a material. In this way the class can familiarize with the behaviour generated by a new action-at-a-distance force and compare it with the other known force of this type, the gravitational one. The attraction and repulsion actions are empirically studied and connected with the existence of positive and negative electric charges. Moreover the Coulomb's law can be studied and compared with the Newton's law of universal gravitation.

The electric field is defined as the electric force per unit charge $(E=F / q)$; a test charge is introduced to deal with the definition of $E$ and to quantify the force the electric field is exerting on it. We decided to do not study at this time the electric field generated by a dot charge, in order to avoid some students' leanings towards binding the electric fields only on this last expression.

Now it is interesting to reveal the historical and methodological implications related to the Millikan's experience. We start proposing the first part of a video prepared by the Physical Science Study Committee (PSSC) [3] on the experimental apparatus of the oil drop experiment. Now it is possible to look at the Physics laws involved in this experience, by proposing to the students the kinematic and dynamic analysis of some tiny oil drop falling in air.

As the characteristics of a motion in a viscous fluid are not included in the usual didactic program, the students are conducted to understand that, in order to perform the Millikan's experiment, it is firstly necessary to deal with the study of the viscous friction and its related consequences on kinematics. Hence an experiment can be developed in order to measure the terminal speed of tiny metallic balls falling inside some transparent soap within a glass cylinder. The students are guided by proper questions towards the recognition of the characteristics of this motion and to understand that, for the first Newton's law, the upwards force due to friction and to the buoyancy must be equal to the weight force the body is subjected to. Moreover it is possible to find a relationship between the different sizes of these bodies and the relied velocities. With the capacity of handling the reviewed concept of inertia and the equations related to the motion in a viscous fluid, the students can now approach the kinematics and the dynamics of a body subjected to a viscous friction, to the Archimedes' law, to the weight force and finally to an electric force too. It could seem that this experience is not connected to the "force and electric field" topic, however in this way the students can understand a wider and global overview about the involved physical phenomena.

The activity proceeds and the experience becomes now the study of a body falling in a viscous fluid within a reduced volume, i.e. in a small space where an electric field is produced between two charged metallic plates. The Leybold-Heraeus 559 41/42 apparatus for the Millikan's experiment was already available in the school laboratory.

The students become involved in the activity because they were asked to understand by themselves how the experiment should be set up. They are allowed to work independently with the instrumentation, to tune the electric force acting on each oil drop and to observe the different behaviours of the drops. After a first time of observation, the students divided in groups are invited to propose a way for the development of the experiment and the quantification of the electric force.

The request of working in team often helps to improve some skills such as the ability to discuss in peer relations, to support one's own thesis and to reasonably criticize a hypothesis deemed incorrect. Furthermore it is often more incisive than a frontal lesson to study in depth and well understand an issue. It is useful for raising new questions about the phenomenon. The questions proposed by teacher can help to do not lose the first purpose of the activity, to keep up with it and to focus the attention on the key points of the experiment

Some of the questions proposed are:

- What is the effect of the electric field on a charged drop? An on a neutral one?

- What is the motion of the oil drops? Is it the same for all of them? Why?

- Where and in which direction does the electric field act? 
After this first observation, the ocular can be connected to a video projector for a better engagement of the whole class. Starting from these questions it is now possible to face the study of the forces and its related field acting on a certain space. The problem can be examined in depth by formalizing some equations that allow the description of the different steps to reach our final scientific purpose: the hypothesized laws and the experimental requirements can be now analysed and verified.

The class concludes the vision of the PSSC video [3] and the numerous and difficult measurements of the fall times of several oil drops are performed in order to measure their speed. In fact a critical point is that a big dataset is necessary to have reliable results about the charge quantization, and thus an appropriate time have to be dedicated to the data collection.

\section{RESULTS}

After introducing the definition of the electric field, the students were asked to read by themselves some materials $[3,4]$ about the oil drop experiment performed by Millikan. Guided by the teacher they were involved in a discussion on the steps required for studying the motion of a particle as a tiny oil drop in air. For instance, without an applied electric field, it is possible to write an equation due to the second Newton's law:

$$
m a=P-F_{A}-F_{V}=\frac{4}{3} \pi r^{3} g\left(\rho_{\text {body }}-\rho_{\text {fluid }}\right)-6 \pi \eta r v_{0}
$$

where $r$ is the radius of the oil drop considered as a sphere, $F_{A}$ is the Archimedes' force, $\rho_{\text {body }}$ and $\rho_{\text {fluid }}$ are the density of the body and of the fluid respectively, $\eta$ is the viscous friction coefficient and $v_{0}$ is the speed of the body. From this equation it is possible to find the expression of the terminal speed of the body, by imposing to zero its acceleration:

$$
v_{0}=\frac{2}{9} \frac{g r^{2}}{\eta}\left(\rho_{\text {body }}-\rho_{\text {fluid }}\right)
$$

Instead if an oil drop is falling for instance within an upwards uniform field the forces acting on it are for the second Newton's law:

$$
m a=P-F_{A}-F_{V}-F_{E}=\frac{4}{3} \pi r^{3} g\left(\rho_{\text {body }}-\rho_{\text {fluid }}\right)-6 \pi \eta r v_{1}-q E
$$

where $q E$ is the product of the electric charge and the electric field on the oil drop. By imposing to zero the acceleration, it is possible to find a new expression of the terminal speed, that becomes:

$$
v_{1}=\frac{2}{9} \frac{g r^{2}}{\eta}\left(\rho_{\text {body }}-\rho_{\text {fluid }}\right)-\frac{q E}{6 \pi \eta r}=v_{0}-\frac{q E}{6 \pi \eta r}
$$

Thus, in the case of an oil drop standing still when a certain electric field and proceeding with a fixed speed $v_{0}$ when switching off the electric field, its electric charge $q$ can be determined by imposing $v_{1}$ to zero. It is possible to find this relationship:

$$
q=\frac{6 \pi \eta r v_{0}}{E}
$$

By repeating several times the measurement with the same electric field it is possible to collect data about the charge values and plot them in a histogram from which the quantization of charge can be distinguished. The greatest common divisor of all values can be calculated for reach a final results comparable with the expected one.

\section{CONCLUSIONS}

If we succeed in catching the complexity and the entirety of the experience, the "quantization" aspect is not the principal goal.

For this purpose it is possible to ask to the class an assessment about the difficulties found during the implementation and the comprehension of the experiment. Moreover we propose to read some sections of the text "I dieci esperimenti più belli" [5], where the beauty and the difficulty of this experience is highlighted, as well as some critical observations about the data collection obtained by Millikan. A discussion on the inference about the existence of the "quantum of charge" was carried on and greatest common divisor of the whole dataset of quantified electric charges was calculated. 
The comprehension of this activity can be verified and evaluated by some oral presentations performed by the students within a team work, by means of power point presentations, video, pictures and description of the whole activity. The students receive some suggestions but they are fully free to use the tools they prefer to exhibit their own work, using proper references and acquired data.

Also in this case a cooperative work can be useful to study in depth the problem, to learn how to properly describe and summarize the experiments and the logical steps followed to reach the results. Moreover it promotes virtuous relationships by educating students to help each other and to share their own skills about mathematics, technology, dialectic.

\section{ACKNOWLEDGEMENTS}

Our project was developed in the context of the Piano Nazionale Lauree Scientifiche, a government programme which funds didactic innovation in Science, Technology, Engineering and Mathematics (STEM) in Higher Education and at Secondary School level.

We want to thank the school technical assistant Riccardo Camoni for the technical support on the recovery of the experiment setup.

\section{REFERENCES}

[1] M. Zani, M. Bozzi, "La fisica tra la scuola secondaria e l'università. Riflessioni e orientamenti", Nuova Secondaria, vol. XXXVI, no. 1, pp. 84-88, 2018.

[2] B. Balossi, M. Bozzi, M. Zani, "Determining an extraordinary constant: a PLS project", ICERI2019 (XII International Conference of Education, Research and Innovation) - Seville (Spain) 1113/11/2019 - Proceedings of ICERI2019 pp. 2202-2207, 2019.

[3] PSSC - Physical Science Study Committee-, "L'esperimento di Millikan" in La fisica secondo il PSSC, Zanichelli Scuola 2005.

[4] P. Marazzini, M.E. Bergamaschini, L. Mazzoni, L'indagine del mondo fisico, Carlo Signorelli editore, 2002.

[5] G. Johnson, I dieci esperimenti più belli, Bollati Boringhieri, 2009. 